\title{
On the using of Acoustic Black Holes for the study of General Relativity
}

\author{
Judismar Tadeu Guaitolini Junior* \\ Universidade Federal do Espírito Santo \\ Centro de Ciências Exatas \\ Programa de Pós-Graduação em Física \\ Av. Fernando Ferrari 514, Vitória, ES, Brasil, CEP: 29075-910 \\ E-mail: judismarecce.ufes.br
}

\section{Flávio Gimenes Alvarenga}

Universidade Federal do Espírito Santo

Centro Universitário Norte do Espírito Santo

Departamento de Ciências Matemáticas e Naturais

Rodovia BR 101 Norte, Km 60, Bairro Litorâneo, São Mateus, ES, Brasil, CEP: 29932-540

E-mail: flavioalvarenga@ceunes.ufes.br

\begin{abstract}
Black holes are bodies that, according to the theory of General Relativity, attract all the matter that comes too close to their interior. Their existence today have been indicated by several observational data, even if indirect. The prediction of the existence of black holes long before the confirmation of observational data of any kind is an example of the very advanced stage in which theoretical physics has presently developed; and even if today there are some observations available, they are still too faible to provide an analysis of all the aspects predicted in theory for the phenomena. This is the reason why it would be interesting to simulate analogous objects, more accessible than real black holes, based on studies of condensed matter: the so-called acoustic black holes. In this work we develop the mathematical formalism for acoustic black holes, and by building the Painlevé-Gullstrand metric we visualize the analogy between the acoustic metric of a fluid at supersonic speed and General Relativity. We can observe that results obtained by General Relativity, such as the existence of an event horizon, can be rescued with the use of this metric for the fluid. In particular, using Killing vectors we get to the concept of surface gravity, and from it we can calculate the temperature of an acoustic black hole, showing that thermodynamic effects such as the existence of Hawking radiation can also be studied with the use these analogue models.
\end{abstract}

4th International Conference on Fundamental Interactions - ICFI2010,

August 1-7, 2010

Viçosa, Brazil

\footnotetext{
*Speaker.
} 


\section{Introduction}

In 1915 Albert Einstein concluded the General Theory of Relativity, a revolutionary theory that stated that all the energy content of a four-dimensional space-time deforms it, the deformation being proportional to the total amount of energy. One of the impressive results that follow is the prediction of the collapse of matter into a very small region of space, called a singularity, giving rise to a black hole.

Just one year after their publication, Karl Schwarzschild obtained the first solution to the equations of General Relativity that contained a black hole. These objects were however viewed with skepticism for decades by the scientific community, until their existence became associated with the death of stars large enough to collapse.The collapse would then be responsible for the creation of bodies that swallow all the matter around them, and from which even light could not escape.

Stephen Hawking, in the 70's, analyzing the relevance of quantum processes for the formation of black holes, noted that they were not exactly black, but emitted particles. At the process of pair production at their surroundings, one of the virtual particles could be absorbed by the black hole, that would then issue another, losing the energy needed for its creation and therefore evaporating.

Surprisingly, William Unruh, working with a model of a fluid moving with a speed exceeding the velocity of sound, showed that there is a region from where phonons cannot escape, in analogy with the event horizons of black holes. Later studies also indicated that Hawking radiation is also present in the fluid, even if too weak to be detected.

In this paper we develop a model of a moving fluid and, from a metric obtained for this medium, we try to recover results for a curved medium, typical of General Relativity. In particular, we will search for results that make this medium analogous to black holes and where we can make predictions about the radiation emitted [1,2]. The current interest of this lies in the fact that the creation of real black holes in laboratories is far from being feasible with the existing technological apparatus, and thus tests of the theory should be effected with the use of auxiliary models, such as those proposed in analogies with condensed matter physics.

\section{Acoustic Black Holes}

We will develop the concept of an acoustic black holes by using the propagation of sound waves in a fluid in motion [3]. If the fluid is barotropic, inviscid and irrotational, then the equation of motion for the velocity potential describing a sound wave is identical to the propagation in a $(3+1)$ dimensional Lorentzian geometry:

$$
\nabla^{2} \psi=\frac{1}{\sqrt{-g}} \partial_{\mu}\left(\sqrt{-g} g^{\mu v} \partial_{\mu}\right) \psi,
$$

and thus the propagation of the sound wave will be governed by an acoustic metric $g_{\mu v}(t, \mathbf{x})$.

To work with the fluid, we use the continuity equation [4]

$$
\frac{\partial \rho}{\partial t}+\nabla \cdot(\rho \mathbf{v})=0
$$

and the Euler equation

$$
\frac{\partial \mathbf{v}}{\partial t}+(\mathbf{v} \cdot \nabla) \mathbf{v}=-\nabla \phi-\frac{\nabla p}{\rho}
$$


defining the velocity potential $\psi$ by

$$
\mathbf{v}=-\nabla \psi
$$

We will now make perturbations in these equations, where we have $\rho_{0}, p_{0}, \psi_{0}$ and $v_{0}$ as background, and and the subscript 1 to indicate the perturbations. By defining the symmetric matrix

$$
f^{\mu v}(t, \mathbf{x})=\left(\begin{array}{rr}
-1 \vdots & -v_{0}^{j} \\
\cdots & \cdots \\
-v_{0}^{i} \vdots & -\left(c^{2}-v_{0}^{2}\right)
\end{array}\right),
$$

with $c$ being the velocity of light, we can write the sound wave equation as

$$
\partial_{\mu}\left(f^{\mu v} \partial_{v} \psi_{1}\right)=0 .
$$

Since the effective geometry of the fluid must obey (2.1), then from (2.5) and (2.6) we obtain $g_{\mu v}$ as

$$
g_{\mu \nu}=\frac{\rho_{0}}{c}\left(\begin{array}{rcc}
-\left(c^{2}-v_{0}^{2}\right) & \vdots & -v_{0}^{j} \\
\cdots & \cdots \\
-v_{0}^{i} & \vdots & \delta_{i j}
\end{array}\right),
$$

and we can also write the line element

$$
d s^{2}=\frac{\rho_{0}}{c}\left[-c^{2} d t^{2}+\left(d x^{i}-v_{0}^{i} d t\right) \delta_{i j}\left(d x^{j}-v_{0}^{j} d t\right)\right] .
$$

By taking the Schwarzschild metric [5], and making the following change of coordinates

$$
t \rightarrow \bar{t}=t \pm\left[4 \operatorname{Mtanh}^{-1}\left(\sqrt{\frac{2 G M}{r}}\right)-2 \sqrt{2 G M r}\right],
$$

or

$$
d t \rightarrow d \bar{t}=d t \pm \frac{\sqrt{2 G M / r}}{1-2 G M / r} d r,
$$

we obtain the Painlevé-Gullstrand line element:

$$
d s^{2}=-\left(1-\frac{2 G M}{r}\right) d \bar{t}^{2} \pm \sqrt{\frac{2 G M}{r}} d r d \bar{t}+d r^{2}+r^{2}\left(d \theta^{2}+\sin ^{2} \theta d \phi^{2}\right),
$$

which we will abbreviate as "P-G metric".

To put the acoustic metric in the same form of the P-G metric we could take $\rho$ and $c$ as constants and $v_{0}=\sqrt{2 G M / r}$, but this assumption is incompatible with (2.2) which was used to derive (2.8).

Nevertheless, if we set $v_{0}=\sqrt{2 G M / r}$ in the acoustic metric, by picking $c$ as a position independent constant (normalized as $c=1$ ), we obtain from (2.2)

$$
\rho \propto r^{-\frac{3}{2}}
$$

and finally

$$
d s_{\text {acoust }}^{2} \propto r^{-\frac{3}{2}}\left[-\left(1-\frac{2 G M}{r}\right) d \bar{t}^{2} \pm \sqrt{\frac{2 G M}{r}} d r d \bar{t}+d r^{2}+r^{2}\left(d \theta^{2}+\sin ^{2} \theta d \phi^{2}\right)\right] .
$$


We can see that the acoustic metric is equal to the P-G metric, less than a conformal factor. This equality allows us to recover from the acoustic metric the concept of an event horizon, that in this case corresponds to a region where the velocity of the fluid exceeds the speed of sound; for example, in the vicinity of a narrowing of the pipe. There, the sound waves which pass through the narrowing cannot return, just as the electromagnetic waves in the black holes of General Relativity cannot return once they cross the event horizon. We can also recover the concept of surface gravity and Hawking radiation [6], since those quantities are conformal invariants.

\section{Surface Gravity and Hawking Radiation}

In differential geometry, a Killing vector field is a field that preserves the metric of a manifold, i.e., they are infinitesimal generators of isometries. The Killing vectors satisfy the equation

$$
\xi_{; \mu}^{v}+\xi_{; v}^{\mu}=0 \text {. }
$$

The event horizon of a black hole is a null hypersurface normal to the Killing vectors, where we can define the surface gravity [7] by

$$
\kappa^{2}=-\left.\frac{1}{2}\left(g^{\rho \mu} \xi_{; \rho}^{v}\right)\left(\xi_{; \mu}^{v}\right)\right|_{\text {surface }},
$$

and interpret it as the acceleration of a static particle near the event horizon, as measured by an observer at infinity. In the case of a diagonal metric, the surface gravity takes the form

$$
\kappa^{2}=-\left.\frac{1}{8}\left[g^{00} g_{r r}\left(-g^{r r} \partial_{r} g_{00}\right)^{2}+g^{r r} g_{00}\left(-g^{00} \partial_{r} g_{00}\right)^{2}\right]\right|_{\text {surface }} .
$$

For the Schwarzschild metric, knowing that the temperature of the radiation emitted by the black hole is given by $T=\frac{\kappa}{2 \pi}$, we have

$$
T=\frac{h c^{3}}{8 \pi k_{B} G M}
$$

valid for black holes in General Relativity. Now, by making the following change of coordinates

$$
d \tau=d t+\frac{\mathbf{v}_{\mathbf{0}} \cdot d \mathbf{x}}{c^{2}-v_{0}^{2}}
$$

in the acoustic metric, we obtain the diagonal form

$$
d s_{\text {acoust }}^{2}=\frac{\rho_{0}}{c}\left[-\left(c^{2}-v_{0}^{2}\right) d \tau^{2}+\left(\delta_{i j}+\frac{v_{i} v_{j}}{c^{2}-v_{0}^{2}}\right) d x^{i} d x^{j}\right],
$$

that does not have mixed terms of space and time coordinates. This shows that the acoustic metric is static.

With this diagonal form, and using (3.3), we can now obtain the surface gravity of the acoustic black hole:

$$
\kappa=\left.\frac{1}{2} \frac{d}{d n}\left(c^{2}-v_{0}^{2}\right)\right|_{v_{0}=c}
$$

and the temperature of its radiation:

$$
T=\left.\frac{\hbar}{4 \pi c k_{B}} \frac{d}{d n}\left(c^{2}-v_{0}^{2}\right)\right|_{v_{0}=c}
$$

where $\frac{d}{d n}$ is the normal derivative of the fluid as it crosses the event horizon. 


\section{Conclusions}

The use of this analogue model has the advantage of being related to the material content in a simple algebraic manner, while in general relativity non-linear differential equations are used.

We can see that the signature of the acoustic metric is $(-,+,+,+)$, as we would expect of a Lorentzian geometry. Thus, by this approach, we succeed in studying the movement of a fluid in parallel to the concepts of curvature on a manifold.

Other successes of the analogy come from the fact that the acoustic metric recovers the kinematic quantities because it is equal to the P-G metric, less than a conformal factor. On the other hand, for one to recover the dynamic quantities, an analogy in two dimensions $[8,9]$ is needed.

Though it is very difficult to measure the temperature of Hawking radiation from acoustic black holes, since it assumes typical values of the order of $10^{-6} \mathrm{~K}$ [3] in water, this measurement is still much more feasible than producing mini relativistic black holes in laboratory. Furthermore, using fluids as liquid helium or Bose-Einstein condensates, it could become easier to determine the temperature fluctuations, once the temperature of the background is closer to $0 K$. In this way, the analogy becomes an important tool, and allows the studying of black hole phenomena without having real access to them.

\section{References}

[1] W. G. Unruh, Experimental Black Hole Evaporation?, Physical Review Letters 46, 1351-1353 (1981)

[2] W. G. Unruh, Notes on Black Hole Evaporation, Physical Review D 14, 870-892 (1976)

[3] M. Novello, M. Visser, G. Volovik, Artificial Black Holes, World Scientific (2002)

[4] M. Visser, Acoustic Propagation in Fluids: an unexpected example of Lorentzian geometry, (1993) [gr-qc/9311028]

[5] L. D. Landau, E. M. Lifshitz, The Classical Theory of Fields, 4th Edition, Pergamon Press (1975)

[6] S. W.Hawking, Density Black Holes and thermodynamics, Physical Review D 13, 191-197 (1976)

[7] P. K. Townsend, Black Holes, (1997) [gr-qc/9707012]

[8] M. Cadoni, Acoustic analogs of two-dimensional black holes, (2004) [gr-qc/ 04010138 ]

[9] M. Cadoni, S. Mignemi, Acoustic analogues of black hole singularities, (2005) [gr-qc/ 0504143 ] 